Assessment of Presence in

\section{Augmented and Mixed Reality}

Presence in Augmented and Mixed Reality

Alexander Toet

TNO, Soesterberg, Netherlands, lex.toet@tno.nl

Tina Mioch

TNO, Soesterberg, Netherlands,

tina.mioch@tno.nl

\section{Simon Gunkel}

TNO, the Hague, Netherlands,

simon.gunkel@tno.nl

Omar Niamut

TNO, the Hague, Netherlands, omar.niamut@tno.nl

Jan B.F. van Erp

TNO, Soesterberg, Netherlands, jan.vanerp@tno.nl

While the sense of presence in VR has been extensively studied, there are currently no scales available to measure the sense of presence in AR and MR. Here we propose a general Holistic Presence Questionnaire (HPQ), that measures presence through the sense of telepresence, internal and external plausibility and perceived behavioral and cognitive affordances in the mediated environment. The HPQ is sufficiently general to measure presence experienced in any type of multisensory (visual, auditory, haptic and olfactory) setting (including VR, AR and MR systems). By using single items to tap into each of the relevant psychological processing levels the $\mathrm{HPQ}$ is comprehensive and efficient. Individual items are sufficiently concise so that their (repeated) application minimally interferes with the experience.

CCS CONCEPTS $\bullet \quad$ Human-centered
computing Human computer interaction
(HCl) Interaction paradigms Mixed / augmented
reality

Additional Keywords and Phrases: augmented reality, mixed reality, virtual reality, presence

\section{INTRODUCTION}

Next to virtual reality (VR), mixed (MR) and augmented $(A R)$ reality are gaining importance in science, education, training and entertainment, affording new ways of interaction and engagement with real and virtual worlds. While VR environments typically replace the real world with a virtual one through immersion of the user's senses, AR and MR supplement the real world with digital information, affording users real-time interaction with co-existing real and virtual elements. The development of effective immersive AR and MR systems requires efficient and reliable measures to assess their user experience (UX).

A key characteristic typically used to quantify the $U X$ of VR is presence: the extent to which one feels situated and able to act in the VR environment. Various methods have been developed to measure the sense of presence in a mediated (possibly virtual) environment $[1,2]$. They can be classified as objective (instrumental) and subjective (perception based) measures. Objective measures include biomarkers (e.g., heart rate, EEG and EMG measures, skin conductance and skin 
temperature), behavioral measures (e.g., gaze behavior, reflexive responses, postural sway), or measures related to social behavior and task performance in the mediated environment [3]. Objective measures are generally costly and complex and have methodological limitations that do not allow their application in all conditions, while their interpretation is not unequivocal. Subjective measures include questionnaires, self-report ratings or interviews. Although post-experience presence questionnaires are typically lengthy, intrusive and time consuming, they are still the preferred method of investigation since they are cheap and easy to administer [1, 4]. However, postexperience questionnaires do not capture state changes during the experience. This may be remedied by using single-item presence scales [5, $6]$, that afford a less disruptive assessment of presence and may therefore be more suitable to be administered during exposure [7]. Single-item presence scales can effectively combine excellent test-retest reliability and sensitivity with good convergent validity (correlation) with more elaborate multi-item questionnaires [5].

While various scales have been developed to measure the sense of presence in VR, there are currently no scales available that generalize across AR and MR. Next to being relevant, sensitive and reliable, such scales should also be convenient and minimally intrusive, to afford repeated application during the experience without breaking the presence illusion [8].

Here we propose a comprehensive general Holistic Presence Questionnaire (HPQ), that uses a single item to tap into each of the relevant processing levels in the human brain: sensory, emotional, and cognitive, behavioral and reasoning. The HPQ measures presence through the sense of telepresence, internal and external plausibility and perceived behavioral and cognitive affordances in the mediated environment. The
HPQ is sufficiently general to measure presence experienced in any type of multi-sensory (visual, auditory, haptic and olfactory) setting (including VR, AR and MR systems).

\section{RELATED WORK}

Research on UX in AR/MR typically only addresses the degree to which virtual objects are experienced as actual objects in the user's physical environment $[9,10]$. However, next to its sensory aspects, the sense of presence also significantly depends on the affective, behavioral, and cognitive aspects of the experience [11]. Most existing presence questionnaires only address the sensory components of a mediated presence experience. An exception is the Virtual Experience Test (VET) [12] that measures both the sensory (audio, visual, haptic) and affective (the user's internal and external perspective), cognitive, active and relational dimensions of a presence experience. However, the VET is designed for the development of VR environments and is not sufficiently general for the evaluation of AR/MR systems. The Augmented Reality Immersion (ARI) questionnaire [13] is specifically designed for AR applications and measures immersion on the levels of engagement, engrossment and total immersion, with subscales of interest, usability, emotional attachment, attention, presence and flow. However, the scale is rather lengthy (41 items), while only few items implicitly tap into each of the relevant psychological processing levels that mediate the sense of presence.

\section{THE HOLISTIC SOCIAL PRESENCE QUESTIONNAIRE}

We present a new holistic presence questionnaire (HPQ) that measures the user's sense of place [14] (i.e., telepresence, plausibility and perceived affordances) in a mediated environment by tapping into each of the five relevant (sensory, emotional, 
cognitive, behavioral and decision making) processing levels for multisensory environmental stimuli as defined in the conceptual framework by [15]. The HPQ extends most existing questionnaires that typically only measure spatial presence (i.e., telepresence and agency; [16]).

At the sensory level, the relevant quality factor is the perceptual or sensory fidelity of the experience, i.e. the extent to which users fail to perceive or acknowledge the fact that (part of) the environment they perceive is mediated (the illusion of non-mediation: $[17,18])$. Hence, there should not be any noticeable distortions in the mediated representation: the visual representation should be photorealistic and of high quality, audio should be undistorted, and system factors like jitter and delay that affect behavioral realism should minimally interfere with the sensory activation and integration. Note that the fidelity of an experience can differ largely between the different sensory modalities. Such inconsistencies can lead to a strong sense of presence in one modality but not in another. At this level, quality features are related to individual sensory channels, such as visual, auditory or tactile features, and may also be linked to the perception via multiple senses in parallel (e.g., audio-visual features; see [19]). Example quality features for the visual channel include color naturalness, sharpness, darkness (of black areas), brightness, contrast, flicker, blur, geometrical distortion, and coding and packet-loss induced degradations such as blocking, freezing, and slicing. Examples for the auditory channel include audio-streaming quality parameters like localization and timbre, and speech-transmission quality features like coloration, noisiness, loudness, or continuity. For systems that address multiple sensory channels simultaneously, relevant features are e.g. balance and synchronism, and an experience assessment should address the extent to which one feels like being in direct contact with the environment (one's impression that one directly sees, hears, feels, or smells the environment). At this level, the experience can be assessed by rating an item like: "The represented environment feels natural" (item 1 in Table 1).

At the affective or emotional level, the relevant quality factor is the internal plausibility or sensory congruity [20] of the experience, i.e. the extent to which users have the feeling that their multisensory input is coherent [21] and agrees (is congruent and consistent) with their mental model (expectations or memories) of the represented environment [20, 22 , 23]. Hence, the relevant quality feature at this level is the semantic consistency and congruency between all sensory signals, and the experience can be quantified by rating an item like: " $M y$ sensations are consistent and agree with the represented environment" (item 2 in Table 1).

At the cognitive level, the relevant quality factor is the external plausibility or environmental and thematic congruity [20] of the experience, i.e. the perceived fidelity [24], realness [3] or illusion that the represented environment is authentic [25] and a place that can actually be visited [26, 27]. At this level, the experience can be quantified by rating an item like: "The represented environment appears real" (item 3 in Table 1).

At the behavioral level, the relevant quality factor is the degree to which the all elements in the environment afford natural behavior without any limitations or restrictions, i.e. the feeling that one can interact with the environment in natural way. At this level, the experience can be quantified by rating an item like: "My interaction with the represented environment feels realistic" (item 4 in Table 1).

At the reasoning level, the relevant quality factor is the degree of realism of the multisensory representation of the mediated environment. An AR/MR environment with a high degree of fidelity and realism is expected to influence one's 
Table 1. The Holistic Presence Questionnaire (HPQ)

\begin{tabular}{clll}
\hline Item & Level & Aspect & Question \\
\hline 1 & Sensory & Fidelity & The represented environment feels natural. \\
2 & Emotional & Int. plausibility & My sensations are consistent and agree with the represented environment. \\
3 & Cognitive & Ext. plausibility & The represented environment appears real. \\
4 & Behavioral & Agency & My interaction with the represented environment feels realistic. \\
5 & Reasoning & Reasoning & My thoughts in the environment feel natural. \\
\hline
\end{tabular}

reasoning in a similar way as its unmediated counterpart. At this level, the experience can be quantified by rating an item like: "My thoughts in the environment feel natural" (item 5 in Table 1).

\section{CONCLUSION}

Presence in AR/MR implies the ability to interact directly and in a natural way with all the elements in one's environment, whether they are real or mediated [28]. Existing presence questionnaires developed for the assessment of VR UX typically do not apply to AR/MR systems [28, 29]. The HPQ is sufficiently general to measure the quality of presence experiences with systems on any position on the reality-virtuality continuum [30]. The $\mathrm{HPQ}$ covers all psychological aspects of MR and AR experiences that are relevant to achieve a convincing feeling of presence, like the feelings that the represented environment is plausible (consistent and realistic), that the interaction with the environment is realistic (both on the sensory and behavioral levels), and that its affords natural reasoning and decision making. By using a single item for each of the relevant psychological processing levels the HPQ is comprehensive and efficient. Individual items are sufficiently concise so that their (repeated) application during an experience will minimally break the experience. Initial validation studies confirm the content and face validity of the HPQ. In future studies we will test its stability, sensitivity, and convergent validity in different multi-sensory (visual, auditory, haptic and olfactory) system (including VR, AR and MR systems) settings.

\section{ACKNOWLEDGMENTS}

This project was partially funded by TNO's Early Research Project 'Social eXtended Reality'.

\section{REFERENCES}

[1] Simone Grassini, \& Karin Laumann. 2020. Questionnaire measures and physiological correlates of presence: A systematic review. Frontiers in Psychology, 11, Article 349. https://doi.org/10.3389/fpsyg.2020.00349.

[2] R. Skarbez, F.P. Brooks, \& M.C. Whitton. 2017. A survey of presence and related concepts. ACM Computing Surveys, 50 (6), Article 96. https://doi.org/10.1145/3134301.

[3] N. Khenak, J. Vézien, \& P. Bourdot. 2020. Spatial Presence, Performance, and Behavior between Real, Remote, and Virtual Immersive Environments. IEEE Transactions on Visualization and Computer Graphics, 26 (12), 3467-3478. https://doi.org/10.1109/TVCG.2020.3023574.

[4] Dimitri Hein, Christian Mai, \& Heinrich Hußmann. 2018. The usage of presence measurements in research: A review. In: Presence: Proceedings of the International Society for Presence Research Annual Conference. The International Society for Presence Research.

[5] S. Bouchard, G. Robillard, J. St-Jacques, S. Dumoulin, M.J. Patry, \& P. Renaud. 2004. Reliability and validity of a singleitem measure of presence in VR. In: The 3rd IEEE International Workshop on Haptic, Audio and Visual Environments and Their Applications, 59-61. https://doi.org/10.1109/HAVE.2004.1391882

[6] Hunter G. Hoffman, Todd Richards, Barbara Coda, Anne Richards, \& Sam R. Sharar. 2003. The illusion of presence 
in immersive virtual reality during an $\mathrm{AMRI}$ brain scan. CyberPsychology \& Behavior, 6 (2), 127-131. https://doi.org/10.1089/109493103321640310.

[7] Dmitry Alexandrovsky, Susanne Putze, Michael Bonfert, Sebastian Höffner, Pitt Michelmann, Dirk Wenig, Rainer Malaka, \& Jan David Smeddinck. 2020. Examining design choices of questionnaires in VR user studies. In: $2020 \mathrm{CH}$ Conference on Human Factors in Computing Systems. Association for Computing Machinery, 1-21. https://doi.org/10.1145/3313831.3376260

[8] C. Hendrix, \& W. Barfield. 1996. Presence within virtual environments as a function of visual display parameters. Presence: Teleoperators and Virtual Environments, 5 (3), 274-289. https://doi.org/10.1162/pres.1996.5.3.274.

[9] Tibert Verhagen, Charlotte Vonkeman, Frans Feldberg, \& Pløn Verhagen. 2014. Present it like it is here: Creating local presence to improve online product experiences. Computers in Human Behavior, 39, 270-280. https://doi.org/10.1016/j.chb.2014.07.036.

[10] Anne R. Smink, Eva A. van Reijmersdal, Guda van Noort, \& Peter C. Neijens. 2020. Shopping in augmented reality: The effects of spatial presence, personalization and intrusiveness on app and brand responses. Journal of Business Research, 118, 474-485. https://doi.org/10.1016/j.jbusres.2020.07.018.

[11] D.B. Chertoff, S.L. Schatz, R. McDaniel, \& C.A. Bowers. 2008. Improving presence theory through experiential design. Presence: Teleoperators and Virtual Environments, 17 (4), 405-413. https://doi.org/10.1162/pres.17.4.405.

[12] D.B. Chertoff, B. Goldiez, \& J.J. LaViola. 2010. Virtual Experience Test: A virtual environment evaluation questionnaire. In: IEEE Virtual Reality Conference 2010. IEEE Press, https://doi.org/10.1109/VR.2010.5444804

[13] Yiannis Georgiou, \& Eleni A. Kyza. 2017. The development and validation of the ARI questionnaire: An instrument for measuring immersion in location-based augmented reality settings. International Journal of Human-Computer Studies, 98, 24-37. https://doi.org/10.1016/j.ijhcs.2016.09.014

[14] Yi-Fu Tuan. 1977. Space and place: The perspective of experience. Minneapolis, Minnesota, USA: University of Minnesota Press.

[15] Eliane Schreuder, Jan van Erp, Alexander Toet, \& Victor L. Kallen. 2016. Emotional responses to multisensory environmental stimuli. SAGE Open, 6 (1), 1-19. https://doi.org/10.1177/2158244016630591.

[16] Tilo Hartmann, Werner Wirth, Peter Vorderer, Christoph Klimmt, Holger Schramm, \& Saskia Böcking. 2015. Spatial presence theory: State of the art and challenges ahead. In M. Lombard, F. Biocca, J. Freeman, W. ljsselsteijn, \& R.J. Schaevitz (Eds.), Immersed in Media: Telepresence Theory, Measurement \& Technology. Springer International Publishing, 115-135. https://doi.org/10.1007/978-3-31910190-3_7

[17] M. Lombard, \& T. Ditton. 1997. At the heart of It all: the concept of presence. Journal of Computer-Mediated Communications, 3 (2), Article JCMC321. https://doi.org/10.1111/j.1083-6101.1997.tb00072.x.

[18] M. Lombard, R.D. Reich, M.E. Grabe, C.M. Campanella, \& T.B. Ditton. 2000. Presence and television: the role of screen size. In: Human Communication Research: Proceedings of the 1995 Annual Conference of the International Communication Association 75-98. https://doi.org/10.1111/j.1468-2958.2000.tb00750.x
[19] Sebastian Möller, Marcel Wältermann, \& Marie-Neige Garcia. 2014. Features of quality of experience. In: S. Möller, \& A. Raake (Eds.), Quality of Experience: Advanced Concepts, Applications and Methods. Springer International Publishing, 73-84. https://doi.org/10.1007/978-3-319-026817_5

[20] T.J. Cahill. 2018. Dimensions of congruity in immersive virtual environments: A framework for the schematic processing of multimodal sensory cues In: 18th Conference of the International Society for Presence Research. International Society for Presence Research.

[21] R. Skarbez, F. Brooks, \& M. Whitton. 2020. Immersion and coherence: Research agenda and early results. IEEE Transactions on Visualization and Computer Graphics, 1-1. https://doi.org/10.1109/TVCG.2020.2983701.

[22] Matthias Hofer, Tilo Hartmann, Allison Eden, Rabindra Ratan, \& Lindsay Hahn. 2020. The role of plausibility in the experience of spatial presence in virtual environments. Frontiers in Virtual Reality, 1 (Article 2) https://doi.org/10.3389/frvir.2020.00002.

[23] W. Wirth, T. Hartmann, S. Böcking, P. Vorderer, C. Klimmt H. Schramm, T. Saari, J. Laarni, N. Ravaja, F.R. Gouveia, F. Biocca, A. Sacau, L. Jancke, T. Baumgartner, \& T. Jäncke. 2007. A process model of the formation of spatial presence experiences. Media Psychology, 9 (3), 493-525. https://doi.org/10.1080/15213260701283079.

[24] A.L. Alexander, T. Brunyé, J. Sidman, \& S.A. Weil. 2005. From gaming to training: a review of studies on fidelity, immersion, presence, and buy-in and their effects on transfer in PC-based simulations and games. In: The Interservice/Industry Training, Simulation, and Education Conference (I/ITSEC). DARWARS Training Impact Group, 114

[25] Stephen B. Gilbert. 2016. Perceived realism of virtual environments depends on authenticity. Presence: Teleoperators and Virtual Environments, 24 (4), 322-324. https://doi.org/10.1162/PRES_a_00276.

[26] M. Slater. 2009. Place illusion and plausibility can lead to realistic behaviour in immersive virtual environments. Philosophical Transactions of the Royal Society of London B, 364, 3549-3557. https://doi.org/10.1098/rstb.2009.0138.

[27] G. Gonçalves, M. Melo, J. Vasconcelos-Raposo, \& M. Bessa. 2020. Impact of different sensory stimuli on presence in credible virtual environments. IEEE Transactions on Visualization and Computer Graphics, 26 (11), 3231-3240. https://doi.org/10.1109/TVCG.2019.2926978.

[28] D. Benyon. 2012. Presence in blended spaces. Interacting $\begin{array}{llll}\text { with } \quad \text { Computers, } 24 & 24 \text { (4), } 226 .\end{array}$ https://doi.org/10.1016/j.intcom.2012.04.005.

[29] Ina Wagner, Wolfgang Broll, Giulio Jacucci, Kari Kuutii, Rod McCall, Ann Morrison, Dieter Schmalstieg, \& Jean-Jacques Terrin. 2009. On the role of presence in mixed reality. Presence: Teleoperators and Virtual Environments, 18 (4), 249-276. https://doi.org/10.1162/pres.18.4.249.

[30] Paul Milgram, Haruo Takemura, Akira Utsumi, \& Fumio Kishino. 1995. Augmented reality: a class of displays on the reality-virtuality continuum. In: Telemanipulator and Telepresence Technologies. SPIE, 282-292. https://doi.org/10.1117/12.197321 